\title{
Novel quantum criticality due to emergent topological conservation law in high- $T_{c}$ cuprates
}

\author{
T.K. Kopeć* \\ Institute for Low Temperature and Structure Research, Polish Academy of Sciences, P.O.Box 1410, 50-950 Wroclaw 2, Poland
}

\begin{abstract}
We argue that in strongly correlated electron system collective instanton excitations of the phase field (dual to the charge) arise with a great degree of stability, governed by gauge flux changes by an integer multiple of $2 \pi$. By unraveling consequences of the non-trivial topology of the charge gauge $\mathrm{U}(1)$ group, we found that the pinning of $\mu$ and the zero-temperature divergence of charge compressibility $\kappa \sim \partial n_{e} / \partial \mu$ defines novel "hidden" quantum criticality on verge of the Mott transition governed by the protectorate of stable topological numbers rather than Landau paradigm of the symmetry breaking.
\end{abstract}

Key words: strongly correlated systems, superconductivity, topological order PACS: 74.20.-z, 74.20.Mn, 74.72.-h

The understanding of the observed doping dependence of $\mu$ in cuprates remains a puzzle. In the case of LSCO, the angle-resolved photoemission spectroscopy studies [1] have shown that in underdoped samples, the chemical potential is pinned above the top of the lower Hubbard band. The photoemission measurements of core levels also shows that $\mu$ does not move with hole doping in the underdoped region. The behavior of $\mu$ is quite peculiar from the viewpoint of the Fermi liquid theory of the metallic states and signals a dramatic reorganization of the electronic structure of cuprates with doping. In cuprates there is clear evidence for the existence of a special doping point in the lightlyoverdoped region where superconductivity is most robust. This indicates that it could be a quantum critical point (QCP) while the critical fluctuations might be responsible for the unconventional normal state behaviour [2]. The resemblance to a conventional QCP is hampered by the lack of any clear signature of thermodynamic critical behavior. Experiments appear to exclude any broken symmetry around this point although a sharp change in transport properties is observed [3].

* e-mail: kopec@int.pan.wroc.pl
We explore Mott transitions from the non-magnetic insulator to a superconductor induced by doping and show that the process is governed by the topological structure of the electromagnetic compact gauge $\mathrm{U}(1)$ group. As a result collective instanton excitations of the phase field (dual to the charge) arise with a great degree of stability, governed by gauge flux changes by an integer multiples of $2 \pi$, which labels topologically ordered ground states. The associated abrupt transition between differnt "vacua" allows us to make link between the unusual behavior of the chemical potential and a novel type of quantum criticality that goes beyond the paradigm of the symmetry breaking.

We consider an effective one-band electronic Hamiltonian on a tetragonal lattice that emphasises strong anisotropy and the presence of a layered $\mathrm{CuO}_{2}$ stacking sequence in cuprates: $H=\sum_{\ell} H^{(\ell)}$, where $H^{(\ell)}=$ $H_{t J}^{(\ell)}+H_{U}^{(\ell)}+H_{\perp}^{(\ell)}+H_{\mu}^{(\ell)}$, where

$$
\begin{aligned}
& H_{t J}^{(\ell)}=-\sum_{\left\langle\mathbf{r r}^{\prime}\right\rangle} t c_{\alpha \ell}^{\dagger}(\mathbf{r}) c_{\alpha \ell}\left(\mathbf{r}^{\prime}\right)+t^{\prime} \sum_{\left\langle\left\langle\mathbf{r r}^{\prime}\right\rangle\right\rangle} c_{\alpha \ell}^{\dagger}(\mathbf{r}) c_{\alpha \ell}\left(\mathbf{r}^{\prime}\right) \\
& +J \sum_{\left\langle\mathbf{r r}^{\prime}\right\rangle}\left[\mathbf{S}_{\ell}(\mathbf{r}) \cdot \mathbf{S}_{\ell}\left(\mathbf{r}^{\prime}\right)-\frac{n_{\ell}(\mathbf{r}) n_{\ell}\left(\mathbf{r}^{\prime}\right)}{4}\right] .
\end{aligned}
$$


Here, $\left\langle\mathbf{r}, \mathbf{r}^{\prime}\right\rangle$ and $\left\langle\left\langle\mathbf{r}, \mathbf{r}^{\prime}\right\rangle\right\rangle$ denotes summation over the nearest-neighbour and next-nearest-neighbour sites labelled by $1 \leq \mathbf{r} \leq N$ within the $\mathrm{CuO}$ plane, respectively, with $t, t^{\prime}$ being the bare hopping integrals $t^{\prime}>0$, while $1 \leq \ell \leq N_{\perp}$ labels copper-oxide layers. The operator $c_{\alpha \ell}^{\dagger}(\mathbf{r})$ creates an electron of spin $\alpha$ at the lattice site $(\mathbf{r}, \ell)$. Next, $S_{\ell}^{a}(\mathbf{r})$ stands for spin operator and $J$ is the antifferomagnetic exchange. Further, $n_{\ell}(\mathbf{r})=n_{\uparrow \ell}(\mathbf{r})+n_{\downarrow \ell}(\mathbf{r})$ is the electron number operator, where $n_{\alpha \ell}(\mathbf{r})=c_{\alpha \ell}^{\dagger}(\mathbf{r}) c_{\alpha \ell}(\mathbf{r})$, respectively; $H_{\mu}^{(\ell)}=-\mu \sum_{\mathbf{r}} n_{\ell}(\mathbf{r})$ and $\mu$ is the chemical potential. The Hubbard term is $H_{U}^{(\ell)}=\sum_{\ell \mathbf{r}} U n_{\uparrow \ell}(\mathbf{r}) n_{\downarrow \ell}(\mathbf{r})$ with the on-site repulsion Coulomb energy $U$, while $H_{\perp}^{(\ell)}=$ $-\sum_{\mathbf{r r}^{\prime}} t_{\perp}\left(\mathbf{r r}^{\prime}\right) c_{\alpha \ell}^{\dagger}(\mathbf{r}) c_{\alpha \ell+1}\left(\mathbf{r}^{\prime}\right)$ facilitates the interlayer coupling, where $t_{\perp}$ is the interlayer hopping with the $c$-axis dispersion $\epsilon_{\perp}\left(\mathbf{k}, k_{z}\right)=2 t_{\perp}(\mathbf{k}) \cos \left(c k_{z}\right)$, while $t_{\perp}(\mathbf{k})=t_{\perp}\left[\cos \left(a k_{x}\right)-\cos \left(a k_{y}\right)\right]^{2}$.

We decouple the Hubbard term $H_{U}$ using the collective variable and $i V(\mathbf{r} \tau)$ conjugate to the local particle number $n_{\ell}(\mathbf{r} \tau)$. Further, we introduce the phase (or "flux") field $\phi_{\ell}(\mathbf{r} \tau)$ via the Faraday-type relation $\dot{\phi}_{\ell}(\mathbf{r} \tau) \equiv \frac{\partial \phi_{\ell}(\mathbf{r} \tau)}{\partial \tau}=\tilde{V}_{\ell}(\mathbf{r} \tau)$ and perform the gauge transformation to the new fermionic variables $f_{\alpha \ell}(\mathbf{r} \tau)$, where $c_{\alpha \ell}(\mathbf{r} \tau)=e^{i \phi_{\ell}(\mathbf{r} \tau)} f_{\alpha \ell}(\mathbf{r} \tau)$. The electromagnetic $\mathrm{U}(1)$ group governing the phase field is compact, i.e. $\phi_{\ell}(\mathbf{r} \tau)$ has the topology of a circle $\left(S_{1}\right)$, so that instanton effects can arise due to non-homotopic mappings of the configuration space onto the gauge group $S_{1} \rightarrow \mathrm{U}(1)$. Therefore, we concentrate on closed paths in the imaginary time $0 \leq \tau \leq \beta \equiv 1 / k_{B} T$ which fall into distinct, disconnected (homotopy) classes labelled by the integer winding number $m_{\ell}(\mathbf{r})[4]$. In the limit of strong (weak) correlations the electron number $n_{e} \equiv\langle\bar{c} c\rangle$ interpolates between topological $n_{b} \equiv$ $\langle m\rangle$ (fermionic $n_{f} \equiv\langle\bar{f} f\rangle$ ) occupation numbers [5]. In the large $-U$ limit $\mu \rightarrow n_{f} U / 2$, so that $n_{e} \rightarrow n_{b}$ and the system behaves as governed entirely by U(1) topological charges which play the role of "quasiparticles". Moreover, due to the frustrated motion of the carriers in the fluctuating bath of $\mathrm{U}(1)$ gauge potentials the actual tight-binding parameters become "dressed" $t_{X}^{\star}=t_{X}\left\langle e^{-i\left[\phi_{\ell}(\mathbf{r} \tau)-\phi_{\ell}\left(\mathbf{r}^{\prime} \tau\right)\right]}\right\rangle$, where $t_{X}=t, t^{\prime}, t_{\perp}$ are the bare band parameters. It is instructive to calculate the charge compressibility $\kappa=\partial n_{e} / \partial \mu$. The result is given in Fig.1 along with the outcome for the superconducting phase boundary. We see the evolution of $\kappa$ with decreasing $n_{e}$, (i.e. hole doping) from the Mott insulator [6] with $\kappa=0($ at $2 \mu / U=1)$ to a point of degeneracy on the brink of the particle occupation change at $2 \mu / U=.5$ where $\kappa=\infty$ at $T=0$. This is also the point on the phase diagram from which the superconducting lobe emanates. It is clear that, the nature of the divergence of $\kappa$ here has little to do with singular fluctuations due to spontaneous symmetry breaking as in the "conventional" phase transition. Rather, this di-

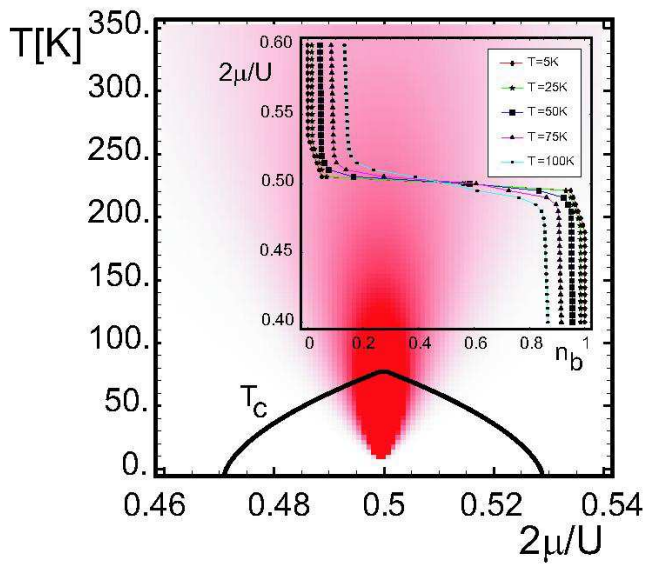

Fig. 1. The superconducting critical temperature $T_{c}$ as a function of the chemical potential $\mu$ together with the density plot of the charge compressibility $\tilde{\kappa} \equiv U \kappa / 2$ for $t^{\star}=0.5 \mathrm{eV}$, $t^{\star \star} / t^{\star}=0.3, t_{\perp}^{\star}=0.01 \mathrm{eV}, J=0.15 \mathrm{eV}$ and $U=4 \mathrm{eV}$. Inset: the chemical potential $\mu$ as a function of the occupation number $n_{e} \approx n_{b}$ for various temperatures $T$. The value of $\mu$ stays within the charge gap as $n_{b}$ changes.

vergent response appears as a kind of topological protection built in the system against the small changes of $\mu$. Further, $\kappa \rightarrow \infty$ implies that the and $\partial \mu / \partial n_{e}$ becomes vanishingly small at $T=0$ which results in the chemical potential pinning (see, inset of Fig.1).

To conclude, topological effects arise as stable, nonperturbative, collective excitations of the phase field (dual to the charge), which carry novel topological characteristics. These are the winding numbers of $\mathrm{U}(1)$ group: $m_{\ell}(\mathbf{r}) \equiv \frac{1}{2 \pi} \int_{0}^{\beta} d \tau \dot{\phi}_{\ell}(\mathbf{r} \tau)$ that become topologically conserved quantities. It is exactly the appearance of these topological charges that render the system protected against small changes of the Hamiltonian's parameters. This novel conservation does not arise just out of a symmetry of the theory (as "conventional" conservation laws based on Noether's theorem) but it is a consequence of the connectedness, i.e. topology of the phase space, related to the topological properties of the associated symmetry group.

\section{References}

[1] A. Ino et al, Phys. Rev. B 62 (2000) 4137.

[2] C.M.Varma, Phys. Rev. B55 (1997) 14554.

[3] T. Ito, K. Takenaka, and S. Uchida, Phys. Rev. Lett. 70 (1993) 3995

[4] L. S. Schulman, Techniques and Applications of Path Integration (Wiley, New York 1981).

[5] T. K. Kopeć, Phys. Rev. B70 (2004) 054518. 
[6] N. F. Mott, Metal-Insulator Transitions (Taylor \& Francis, London, 1990). 